\title{
Time-sharing SKED and OS/8: A new version
}

\author{
ARTHUR G. SNAPPER \\ Western Michigan University, Kalamazoo, Michigan 49008
}

\begin{abstract}
A new time-sharing SKED program has been developed to simultaneously conduct up to 12 behavioral experiments while the $0 \mathrm{~S} / 8$ operating system is used in the "background." The new system provides improved control over the SKED program while allowing the experimenter to develop new programs or analyze data during the experimental session. A minimum of $16 \mathrm{~K}$ of memory and an $0 \mathrm{~S} / 8$ device is required to use this system.
\end{abstract}

SKED was originally designed in 1966 (Snapper, Knapp, Kushner, \& Kadden, Note 1) to simplify the programming of complex operant experiments and data acquisition schemes for a PDP-8 computer with a $4 \mathrm{~K}$ of memory and with paper-tape peripherals. Over the years, the system has been expanded to permit the use of massstorage devices and the $\mathrm{OS} / 8^{1}$ operating system. Although OS/8 SKED provides very rapid translation of state diagrams into working programs (Snapper, 1975), it is still necessary to reserve computer time for programming purposes. Furthermore, extensive data analysis may require even more computer time on a daily basis than does designing new experiments or even conducting current research. It is time that experimenters who learn to utilize the full capacity of SKED to conduct as many as 12 simultaneous experiments soon discover that more time is used to manage and analyze data on the compu. ter than is used to acquire it in the first place.

Two solutions have been proposed to reduce the competition for computer time by data acquisition and data analysis programs. The first strategy involves acquiring a second computer for programming and data analysis, thus freeing the first computer for dedication to the data acquisition task. This solution has become more feasible with the continued reduction in the cost of computer systems, but it involves acquiring a second OS/8 device for the transfer of data and programs between the two computers. This solution would be practical if both computers possessed an OS/8 device with removable media such as DECtape, disk-cartridge, cassette, etc., so that the data acquired by the first computer can be processed on the second. It might also be practical to directly interconnect the computers so that the data acquisition system could transmit data directly to the data processing system. However, this solution would also require the data analysis computer to be dedicated to the experimental session, since it would need to acquire the data while it was being generated. Therefore, it seems

This work was supported in part by Research Scientist Development Award K2-MH-70483 from the National Institute of Mental Health. likely that this method would also be more convenient if both computers had OS/ 8 devices.

Although this solution is possible, it is also expensive, since two computers are not yet cheaper than one. Furthermore, once one acquires a second computer there is a certain temptation to acquire a second interface and to proceed to double the capacity of the laboratory to conduct research. (This may be a corollary to one of Parkinson's laws.) It is usually easier to justify the acquisition of the second computer on the basis of its capacity to produce experiments rather than for its increased convenience to the experimenter. Laboratories that go through this experience soon discover that the quantity of data analysis has at least doubled. The increased quantity of data requires better data reduction and data analysis schemes just to prevent the laboratory from being overwhelmed by the mass of numbers produced on a daily basis.

An alternative solution that is less expensive and which also can permit simultaneous data analysis and data acquisition involves obtaining enough memory for a single computer so that both SKED and data analysis programs can be in the computer at the same time. Although SKED must respond to each clock tick (once every $10 \mathrm{msec}$ ), the PDP-8 is fast enough to complete its processing of the tick in less than $10 \mathrm{msec}$, leaving time for data analysis until the next clock tick.

The second solution would be ideal if it permitted the use of OS/8 programming in the background, while SKED continued to operate with the highest priority, responding to each clock tick every $10 \mathrm{msec}$. Although it might seem to be a simple programming problem to merge OS/8 with the SKED software given the availability of enough memory for both functions, the interrupt nature of SKED and the PDP-8 introduces a severe complication. OS/8 was originally programmed to run in Field 0 and the initial $8 \mathrm{~K}$ of memory, although many OS/8 programs can utilize any available additional fields. The SKED system, however, must run in Field 0 since the interrupt feature of the PDP-8 requires the first few locations in Field 0 for its operation. Therefore, SKED must retain Field 0, while OS/8 must be moved to other 
fields. The job of moving OS/8 to new fields would be a massive reprogramming task involving redesigning all of the $30 \mathrm{OS} / 8$ programs for this purpose. While it is possible to undertake this project, a rough estimate of work involved suggests that an estimated expenditure of several man years by an experienced programmer would be necessary.

Digital Equipment Corporation has developed an alternative method for running $\mathrm{OS} / 8$ in other memory fields while Field 0 is utilized for real-time programs. This new system, called RTS- 8 , $^{\prime}$ uses a hardware feature called "the trap" to detect instructions referencing fields of operation in OS/8. The trap is an interrupt device that will prevent the trapped instruction from operation while preserving all current status information about the program calling the trap, so that a special program can adjust the instruction for the new OS/8 fields. The RTS8 program uses the trap so that $\mathrm{OS} / 8$ will operate with the computer's interrupt mechanism enabled. There also is a priority arrangement in RTS- 8 so that different devices, such as the SKED clock, can be afforded priority over the background OS/8. The present report describes a new SKED that uses RTS-8 to provide for a background OS/8 while SKED is controlling up to 12 foreground stations.

\section{USING TIME-SHARING SKED}

\section{Hardware Requirements for RTS-8}

The time-sharing SKED system needs at least $16 \mathrm{~K}$ of memory and one of the following OS/8 peripherals: DF32, RK8, RK8E, TC8, or the flexible disk. It will not operate with the TD8E or any other OS/8 system that cannot be interrupted during data transfers. The RTS- 8 and SKED software utilize Field 0. OS $/ 8$ can run in Fields 1 and 2. Field 3 is then available for state tables. With $20 \mathrm{~K}$ of core, the user can request Field 4 for additional state table storage or he can request a $12 \mathrm{~K}$ OS/ 8 background system. We find $24 \mathrm{~K}$ of core permits $12 \mathrm{~K}$ for $\mathrm{OS} / 8$ (thus permitting batch programs) and $8 \mathrm{~K}$ for state tables. The $8 \mathrm{~K}$ state table capacity is sufficient to run 12 stations in my laboratory. The user could assign additional core either to state tables or to $\mathrm{OS} / 8$ as his needs dictate.

The new run-time system differs from the older OS $/ 8$ version in a number of ways. RTS- 8 was designed to require two terminals, one for OS/8 and one for the timesharing monitor. However, for SKED users the only need for a terminal for real-time applications is to load state tables, print data, initiate creation of input files, and stimulate responses to start or stop sessions. Usually only a few minutes are needed on an intermittent basis at the beginning or end of fairly lengthy experimental sessions. The rest of the time, while the subjects are working, is available for programming. Therefore, it seemed reasonable to use a single terminal and to develop an OS/8 background program to monitor SKED.
Thus, the monitor could be like any other OS/8 program when it was needed at the beginning of sessions. At other times, OS $/ 8$ could be used to develop programs or analyze data. For this reason, all Teletype routines, formerly in SKED, were removed and incorporated in an OS $/ 8$ program called MON. Since the OS/ 8 area is at least $8 \mathrm{~K}$, the capabilities of the monitor functions could be increased significantly over the previous versions which were included in SKED and which previously competed for space with state table capacity.

\section{New Capabilities of the SKED Monitor}

A number of features of the monitor have been improved due to the new structure of time-sharing SKED.

Loading state tables. The first and most important of these features concerns the input of state tables. In the older OS/8 run-time system, only nine input files could be specified in advance (although an F3 has been developed to overcome this restriction). In the new system, the input routine can be called at any time to specify new state tables without interfering with stations that are already running.

Saving data files at the end of the session. The same advantage of unlimited access to $\mathrm{OS} / 8$ routines permits complete Teletype control over data files. During or at the end of the session, a named data file can be created, and data collected by any station can be saved on the $\mathrm{OS} / 8$ device. Any number of files can be created at any time just by specifying them on the terminal device.

Storing data files by state table requests. The earlier OS/8 SKED permitted the storage of data on the OS/8 device whenever a storage request occurred in the state table. The only restriction was that all stations had to store data on one output file. After the session was over, it would require considerable computer time just to separate data from different experiments into separate files for further analysis. Also, the old system required the experimenter to reserve storage space for the data by requesting twice the number of counters needed for the recording. The new system permits separate output files to be recorded simultaneously by as many as 12 different stations. The experimenter must include the name of the file to be created and the number of blocks of data to be recorded. Each block can contain 248 recording counters plus the standard light identification numbers. If no extension is specified, SKED will automatically create an extension based upon the station containing the state table. In this way, up to 12 different data files may be generated simultaneously. Recording a block of data will then be initiated by a simple state table request which can be contingent on response or time statements. If the experimenter has not specified enough room for the data in his file opening statement, the SKED program will terminate when the file is completely filled. It is important to note that the run-time system can be recording data, while OS/ 8 is also using the system in the background, manipulating files at the same time without 
much interaction between the two operations. If recording on-line is too frequent, however, the background program may proceed very slowly.

Printing data. A routine has been added to the monitor to print specific recording counters of one station. Earlier versions require printing all of the counters assigned to the station. The new version asks for the number of the first and the last counter to be printed. It also does not print the identifiers, since they are primarily designed for use by data analysis programs. The print routine, then, permits the experimenter to monitor the number of trials or reinforcements that have occurred in a station during the session without having to print all of the counters.

Identifiers. The station identifiers for experiment, group, and subject numbers are created by a new OS $/ 8$ program called IDENT. They are then stored as an OS $/ 8$ binary file that may be read by the monitor and recorded along with the data. The system data, the station number, and the number of recording counters in the file are still automatically created by SKED.

Status. A new function has been added to report the stations that are currently active. This is useful for determining when the session has ended for various stations.

Batch. The program is easier to use under the control of a batch program in that arguments such as input and output files can be passed automatically to SKED by means of the monitor program and BATCH.

In summary, the new time-sharing SKED monitor improves the conveniences and flexibility of SKED. Several restrictions of OS/8 SKED concerning the number of input and output files open at the same time have been circumvented in the time-sharing system. Furthermore, the basic capability of programming with OS/8 at the same time that sessions are running has been added. The cost of time-sharing SKED to the OS/8 user is an additional $8 \mathrm{~K}$ of memory and the RTS- 8 software system. The OS/8 background system is somewhat slower in operation than the normal OS/8. For the SKED user, the major difference in speed of response is most evident in the EDITOR program, which is very slow in the background. However, the powerful TECO editor is not noticeably slower in the background than in the foreground. The major reason for the reduction in speed of the EDITOR is the large number of data field instructions, each of which activates the hardware trap.

\section{New Developments in SKED}

It has been my custom to announce planned changes and developments in SKED for the next year at each of these conferences. We are currently at work on a change in the notation and an associated SKED program. This development will include a completely new compiler, and SKED built around the new notation. Briefly, the contemplated changes deal with the treatment of variables and recording counters in state diagrams and tables. Currently, the $F 1$ and $F 2$ functions and gating provide a minimum degree of flexibility in terms of online data processing. These functions were primarily designed to simplify the programming of histograms. For this purpose, they permitted the setting of variabies to an initial value and the linear incrementation, or decrementation, of these values by a constant to a prespecified limit. Planned changes include incorporating LET statements of the sort used in BASIC, and the addition of multiplication and division operators. Furthermore, we plan to include an IF statement with complete generality of operators as in BASIC. The additional changes contemplated include the simplification of the format for some standard F3 machine language programs. It has not yet been determined whether these changes will be possible without the requirement of a hardware multiply and divide unit to reduce the amount of time necessary for the new operators. We currently are attempting to make the new version of the software "upward compatible" with the old, so that state tables usable in the current system will operate in the newer version. We expect to announce the availability of the new version by the conference next year.

\section{REFERENCE NOTE}

Snapper, A. G., Knapp, J. Z., Kushner, H. K., \& Kadden, R. M. A notation system and computer program for behavioral experiments. Paper presented at the meeting of the Digital Equipment Computer Users Society (DECUS), New York, June 1967.

\section{REFERENCE}

SNAPPER, A. G. A new OS/8 SKED. Behavior Research Methods \& Instrumentation, 1975, 7, 233-238.

\section{NOTE}

1. Copyright Digital Equipment Corporation, Maynard, Massachusetts. 